\title{
Think biology!
}

Jacques Menetrey

Published online: 2 October 2010

(C) Springer-Verlag 2010

Function creates organs

Claude Bernard (1813-1878)

Over the last two decades, surgical techniques for soft tissue repair and reconstruction have dramatically improved, but changes in technique have been very frequent especially for ACL reconstruction. Surprisingly, the majority of these changes have more often been "marketdriven" rather than scientifically supported. However, you can argue that the ACL double bundle reconstruction technique has recently allowed for the "re-discovery" of the anatomical landmark of the native ACL. While this is correct, our outcomes have remained fairly steady over the past 15 years $[1,2]$.

From the earliest days of orthopaedic sports medicine, and certainly influenced by the history of general orthopaedics, surgeons have been thinking in a mechanistic way; they want things fixed in a solid manner. Indeed, if one wants his athletes to rapidly return to sports activities, one needs to adhere to solid principles. And this is correct even from a biological perspective, because the only evidence we have at the present time as regards the healing process is that it is directly dependent upon the mechanical environment [3]. However, we must realise that from the first encounter with our patient in the waiting room to complete healing, we are dealing with a living organism with its subtle, genuine and complex specificity. Naturally, biology is everywhere, particularly in surgery and even more specifically in orthopaedics sports medicine, where we deal

J. Menetrey $(\bowtie)$

Unité d'Orthopédie et Traumatologie de Sport,

Service de Chirurgie Orthopédique et Traumatologie

de l'Appareil Moteur, HUG, Rue Gabrielle-Perret-Gentil 4,

1211 Genève 14, Switzerland

e-mail: jacques.menetrey@hcuge.ch with diverse tissues such as cartilage, subchondral bone, meniscus, rotator cuff, tendon and ligament, particularly the anterior cruciate ligament. These tissues have low biological capacity for healing and are often in a diminished state when the surgeon first operates the patient.

Biology is everywhere and comes into play when the first indication for any procedure is discussed with the patient (Think individualised treatment). In this regard, we have no help from the biology side to distinguish between patients with a low or high risk for surgical complications and to determine who is a slow or fast healer. We know very little about the expression of the particular genes that define fast, slow or no healing, and even less about the genetic profile, which characterises those traits [4]. Up until the present time, physicians have been more interested in other factors such as age, presence of diabetes and other co-morbidities, previous surgery, and drug or cigarette consumption, but these factors are not sufficient to soundly answer the frequently asked question about outcome and prognosis. Genetics in orthopaedics is a promising field of investigation in the coming decades.

Following a lecture on a particular surgical technique, a non-surgeon colleague raised his hand and commented a bit aggressively, "with your surgery you only make the biological environment worse and profoundly disturb the healing process in such a way that is certainly more detrimental than it is beneficial to complete recovery". I must say that he was not completely wrong because surgery certainly has a major impact on the "treated" tissue, but who speaks of surgery speaks of the surgeon. And from this "orthobiology" perspective, the surgeon does matter at least as much as the patient. This is probably why, regardless of the surgery, the surgeon is such a substantial confounding factor in clinical studies. As surgeons, we should know not only the anatomy of the tissues we 
encounter but also their biological characteristics, and as well their mechanism and specific capacity for healing. We should never forget that we are only a guest in the operating field and our performance should be as much biologically oriented as mechanical. Be smooth and remember that arthroscopy is a "gliding sport." Respect and honour the fat pad and don't let yourself, the resident or the fellow wildly shave the anterior compartment of the knee! Don't overtension your ACL graft as this is detrimental to the ligamentisation process [5]. Don't dig too aggressively into subchondral bone as you will impede all cartilage repair techniques (microfracturing is certainly the simplest and the most surgeon-dependent technique). The surgeon should be more like a gardener rather than a carpenter.

In the post-operative phase, biology is obviously a "must" in order to move towards uneventful healing. At this point, biology rhymes with personality and activity. Thus far, we have had a relative boorish and empirical approach to track these two factors, and we still lack appropriate biological parameters to objectively follow the early phase of healing. It would be ever so advantageous to have biological marker to monitor ACL ligamentisation or rotator cuff incorporation. Activity, or in other words motion, is a potent stimulator of biology. Function creates the organ, but this function must be appropriate to the state of healing. Put in optimal condition, biology can a lot and we tend to forget it since, for obvious reason, most of our studies are made at time zero. In any event, it is an evolving system that shows incredible adaptive properties to non-optimal situations, which may also generate an inadequate or hypertrophic response, and as well even seemingly recreate a double-bundle ACL (see Fig. 1). Having said that, it is mandatory to monitor the postoperative period with the tools we currently have available. Indeed, one can dramatically change patient outcome by simply considering the degree of inflammation and the activity level at different points in time. Out of "business" considerations, this rule is also true for professional athletes and superstars.

Biology has made his way into the orthopaedic community thanks to cartilage repair and mesenchymal stem cell technology; efforts to stimulate tendon and ligament healing; tissue engineering for the meniscus; and many other developments. Nevertheless, I am always surprised at our meetings by the low attendance at research sessions.

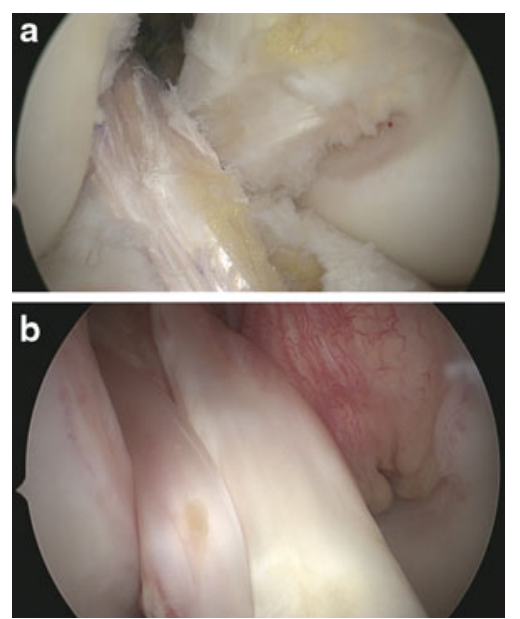

Fig. 1 a Basketball player, 29-year-old, ACL reconstruction with BPTB, single bundle, interference screw fixation. b Same patient 5 years later, second arthroscopy for lateral meniscus tear following hazardous landing while playing basketball

Apart from specific textbooks, this is where one learns how tissue basically functions, and often one can immediately implement some of the fascinating knowledge available at these sessions.

The modern orthopaedic surgeon should be trained, conduct research and be constantly educated in this living field. Think biology!

\section{References}

1. Laxdal G, Kartus J, Ejerhed L et al (2005) Outcome and risk factors after anterior cruciate ligament reconstruction: a follow-up study of 948 patients. Arthroscopy 21:958-964

2. Lewis PB, Parameswaran AD, Rue JP, Bach BR Jr (2008) Systematic review of single-bundle anterior cruciate ligament reconstruction outcomes: a baseline assessment for consideration of double-bundle techniques. Am J Sports Med 36:2028-2030

3. Menetrey J, Duthon VB, Laumonier T, Fritschy D (2008) Biological failure of the anterior cruciate ligament. Knee Surg Sports Traumatol Arthrosc 16:224-231

4. Ward BD, Furman BD, Huebner JL, Kraus VB, Guilak T, Olson SA (2008) Absence of post-traumatic arthritis following intraarticular fracture in the MRL/MpJ mouse. Arthritis Rheum 58:497-503

5. Yoshiya S, Andrish JT, Manley MT, Bauer TW (1987) Graft tension in anterior cruciate ligament reconstruction. An in vivo study in dogs. Am J Sports Med 15:464-470 